PAPER

\title{
Tau gene and Parkinson's disease: a case-control study and meta-analysis
}

\author{
D G Healy, P M Abou-Sleiman, A J Lees, J P Casas, N Quinn, K Bhatia, A D Hingorani, N W Wood
}

See end of article for authors' affiliations

....................

Correspondence to: Dr Daniel G Healy, Department of Molecular Neuroscience, Institute of Neurology, Queen Square, London WCIN 3BG, UK n.wood@ion.ucl.ac.uk

Received 19 August 2003 Revised 21 November 2003 Accepted5December 2003

J Neurol Neurosurg Psychiatry 2004;75:962-965. doi: 10.1136/jnnp.2003.026203

...................

\begin{abstract}
Objective: To investigate whether the tau $\mathrm{H} 1$ haplotype is a genetic risk factor in Parkinson's disease and to report a meta-analysis on all previously published data

Methods and results: In a sample of 580 patients with Parkinson's disease and 513 controls there was an increased risk of Parkinson's disease for both the tau HI haplotype ( $p \leqslant 0.0064$; odds ratio (OR) 1.34 (95\% confidence interval (CI), 1.04 to 1.72)) and the $\mathrm{H} 1 \mathrm{H} 1$ genotype ( $\mathrm{p} \leqslant 0.0047$; OR 1.42 (1.1 to 1.83)). Under a fixed effect model, the summary OR for this showed that individuals homozygous for the $\mathrm{H} 1$ allele were 1.57 times more likely to develop Parkinson's disease than individuals carrying the $\mathrm{H} 2$ allele $195 \% \mathrm{Cl}$ 1.33 to $1.85 ; \mathrm{p}<0.00001$ ). The population attributable risk for the tau variant, for the main comparison of $\mathrm{H} 1 \mathrm{H} 1$ against $\mathrm{H} 2$ carriers, was $24.8 \%$ for all studies combined.

Conclusions: Homozygosity for the tau $\mathrm{Hl}$ is associated with an increased risk of Parkinson's disease. This adds to the growing body of evidence that common genetic variation contributes to the pathogenesis of this disorder.
\end{abstract}

$\mathrm{T}$ here is good evidence implicating the tau gene (MAPT) in the pathogenesis of Parkinson's disease. A large, family based genome-wide search of late onset cases reported linkage to the tau locus on chromosome $17 \mathrm{q},{ }^{1}$ and, while no tau gene mutations have been found in typical Parkinson's disease, mutations have been reported in cases of frontotemporal dementia with parkinsonism on chromosome 17 (FTDP-17). ${ }^{2}$

Recent evidence suggests that tau can co-aggregate with $\alpha$ synuclein in the Lewy body, ${ }^{3}$ and Giasson $e t$ al ${ }^{4}$ have shown that tau and $\alpha$ synuclein interact to promote and propagate the polymerisation of each component into fibrils. In addition, tau aggregation-sometimes in the absence of Lewy body pathology - has been demonstrated in autosomal recessive Parkinson's disease caused by parkin gene mutations. ${ }^{5}$

Nine genetic case-control association studies have evaluated the association between the tau gene and Parkinson's disease. $^{6-14}$ However, the results from these individually underpowered studies have failed to resolved this question. We have therefore carried out a new and larger case-control association study assessing tau and Parkinson's disease in the British population. We then combined our results with all previous studies in a meta-analysis to provide a more precise estimate of the tau-Parkinson's disease association.

\section{METHODS}

The project was approved by the joint research ethics committee of the Institute of Neurology and the National Hospital for Neurology and Neurosurgery (NHNN), London, UK.

\section{Study population}

In all, 580 patients with Parkinson's disease and 513 controls participated in the study. The Parkinson's disease cohort consisted of 193 sporadic cases, 263 unrelated patients with onset of disease before the age of 50 years (YOPD), and 124 index familial cases with one or more relative also affected by Parkinson's disease (FPD). The following were excluded from the study:

- familial cases where the family history was clearly autosomal dominant or recessive;

- individuals harbouring parkin (PARK2) gene mutations $(\mathrm{n}=12)$;

- individual carriers for pathogenic DJ-1 mutations $(\mathrm{n}=2)$.

However, not all cases were screened for mutations in parkin and only 400 cases were screened for mutations in DJ-1. Sixty patients had pathologically proven Lewy body Parkinson's disease.

All living patients were diagnosed clinically by at least one consultant neurologist with an interest in movement disorders and in accordance with the modified Queen Square Brain Bank criteria for Parkinson's disease. ${ }^{15}$

The control cohort consisted of unrelated patient spouses $(\mathrm{n}=168)$, healthy volunteers $(\mathrm{n}=66)$, and unrelated patients seen at the NHNN for other neurological disorders including peripheral neuropathy $(\mathrm{n}=104)$, Huntington's disease $(n=69)$, ataxia $(n=41)$, and epilepsy $(n=65)$. All control subjects had been assessed by a consultant neurologist, and none had parkinsonism.

Patients and controls were all white British and lived in south east England. Both groups were matched for age (Parkinson's disease, 53.8 years (age of onset); control, 56.1 years) and sex (male 52\% $v 49 \%$ ). A similar proportion of patients and controls were in the age categories $<45$ years, 45 to 65 years, and $>65$ years). Neither group had previously participated in studies relating to the tau gene.

\section{Genotyping}

The tau haplotype was inferred as previously described by assessing for the presence or absence of a 238 base pair deletion in intron 9 of the H2 haplotype, ${ }^{16}$ with presence defining $\mathrm{Hl}$ and absence defining H2. Genotyping was conducted blinded to the clinical status.

Some reports included in the meta-analysis predate Baker et al, ${ }^{16}$ showing the extended $\mathrm{Hl}$ and $\mathrm{H} 2$ tau haplotypes.

Abbreviations: FPD, familial Parkinson's disease; LD, linkage disequilibrium; PSP, progressive supranuclear palsy; YOPD, young onset Parkinson's disease 
These reports used an intronic dinucleotide repeat (IVS 9), comprising five alleles (A0, A1, A2, A3, and A4 alleles). The $\mathrm{A} 0, \mathrm{Al}$, and $\mathrm{A} 2$ alleles constitute $\mathrm{H} 1$ haplotype, while $\mathrm{A} 3$ and A4 constitute the $\mathrm{H} 2$ haplotype.

\section{Statistical analysis}

For the case-control study, tau haplotype and genotype frequencies were calculated using the standard Pearson $\chi^{2}$ test. To test for association between tau $\mathrm{Hl}$ and Parkinson's disease, univariate and multivariate odds ratios (OR), the corresponding 95\% confidence intervals (CI), and probability (p) values were obtained by logistic regression methods.

We then carried out a meta-analysis which included our data. Two electronic databases (Medline and Embase) were searched up to August 2003 for all studies evaluating the tau $\mathrm{Hl}$ and Parkinson's disease. For inclusion, the following criteria had to be fulfilled:

- the study was a case-control design (retrospective or nested);

- the subjects were unrelated and white;

- the diagnosis of Parkinson's disease was made clinically or pathologically confirmed;

- the study involved examining the associations between Parkinson's disease and tau Hl.

Studies were excluded if they were reported only in abstract form, or if genotype or haplotype frequency was not reported.

Terms used for the search were both MeSH terms and text words: "tau" and "Parkinson's disease" in combination with "genetic," "haplotype," "Hl," and "association study." The search results were limited to "human" and "English language." We searched for any additional studies in the references of all identified publications. Two investigators independently reviewed study eligibility, and inconsistencies were resolved by consensus.

As in the case-control study, the a priori hypothesis in the meta-analysis was that $\mathrm{Hl}$ homozygosity compared with carriers of $\mathrm{H} 2(\mathrm{H} 1 \mathrm{H} 2+\mathrm{H} 2 \mathrm{H} 2)$ would be associated with an increased risk of Parkinson's disease. In a separate analysis, odds ratios for other genetic models were also calculated. We calculated a fixed effect and a random effect summary odds ratio and $95 \%$ confidence interval for the association. Fixed effect summary odds ratios were calculated using the Mantel-Haenszel method, ${ }^{17}{ }^{18}$ and the DerSimonian and Lair method was used to calculate random effect summary odds ratio. ${ }^{19}$ The DerSimonian and Laird $Q$ test was also used to evaluate the degree of heterogeneity between studies, and $\mathrm{I}^{2}$ was used as a measure to describe the percentage of variability in point estimates resulting from heterogeneity rather than sampling error. ${ }^{20} \mathrm{~A}$ funnel plot was used to assess for publication bias. ${ }^{21}$ In addition, the influence of individual studies on the summary odds ratio was evaluated by reestimating and plotting the summary odds ratio in the absence of each study.

The population attributable risk-reflecting the proportion of Parkinson's disease in the population that can be attributed to a particular risk factor-was calculated for the tau variant using the following formula:

$100 \times$ [prevalence $(\mathrm{OR}-1) /$ prevalence $(\mathrm{OR}-1)+1]$.

For this calculation we used the OR derived from the fixed method from the meta-analysis, and the proportion of the total population exposed to the putative risk (that is, $\mathrm{Hl}$ ) was calculated from genotype frequencies in the control group. Data were analysed using the Review Manager software (version 4.2) from the Cochrane Collaboration 2003.

We also undertook a secondary analysis in which we attempted to control for a possible misdiagnosis of progressive supranuclear palsy (PSP) in our Parkinson's disease population. We made the assumption, as reported by another study from this institution, ${ }^{22}$ that $6 \%$ of our patients had PSP but had been clinically misdiagnosed as Parkinson's disease. We also assumed that $87.5 \%$ of those PSP patients were $\mathrm{H} 1 \mathrm{Hl}$ and $12.5 \% \mathrm{H} 1 \mathrm{H} 2$, based on other studies. ${ }^{16}$ We therefore randomly removed 35 patients with Parkinson's disease $(6 \%), 31$ with the H1Hl genotype $(87.5 \%)$, and four with the $\mathrm{H} 1 \mathrm{H} 2$ genotype $(12.5 \%)$.

\section{RESULTS}

\section{Case-control study}

Analysis of our control population showed no deviation from Hardy-Weinberg equilibrium $(p=0.54)$, but our Parkinson cohort was not in Hardy-Weinberg equilibrium $(p=0.03)$.

We observed the tau $\mathrm{Hl}$ haplotype in $74.8 \%$ of our controls and $79.7 \%$ of our overall Parkinson's disease population. This result was highly significant (OR 1.32 (95\% CI, 1.10 to 1.62 ); $\mathrm{p}=0.006)$.

Using a recessive genetic model comparing the $\mathrm{HlHl}$ genotype with $\mathrm{H} 1 \mathrm{H} 2$ plus $\mathrm{H} 2 \mathrm{H} 2$, subjects homozygous for $\mathrm{Hl}$ were 1.43 times more likely to develop Parkinson's disease than carriers of the $\mathrm{H} 2$ allele (95\% CI, 1.12 to $1.82 ; \mathrm{p}=0.004$ ). This increased risk remained significant after adjustment, as outlined above, for potential PSP misdiagnosis (OR 1.34 ( 1.04 to 1.70$) ; \mathrm{p}=0.02)$. In fact we estimate that a PSP misdiagnosis rate of $26 \%$ would be required for the odds ratio to cross 1.0 .

When we analysed YOPD separately, the Hl effect remained significant (OR 1.38 (1.06 to 1.80$) ; \mathrm{p}=0.01$ ). We observed a particularly strong association between the $\mathrm{HlHl}$ genotype and YOPD (OR 1.6 ( 1.2 to 2.2 ); $\mathrm{p}<0.004$ ), although the $95 \%$ CI for all cases of Parkinson's disease and the YOPD group overlap. There was no association in the FPD group $(p=0.4)$. This may reflect the relatively small number of patients in this subgroup $(n=124)$, or may be accounted for by unidentified single gene mutations such as PARK2. These data are displayed in table 1.

\section{Meta-analysis results}

The primary search identified 10 potentially relevant articles, of which seven-including the present case-control studymet the selection criteria. Of the three articles excluded, one was a family based study, ${ }^{14}$ another did not provide enough data, ${ }^{11}$ and the third was not reported as a full text paper. ${ }^{12}$ No prospective, cohort, or nested case-control studies were identified.

Overall, the genotype frequency for individuals homozygous for the $\mathrm{Hl}$ allele in the control population was $57.9 \%$ (95\% CI, $52.5 \%$ to $63.5 \%$ ); for heterozygous individuals $(\mathrm{H} 1 \mathrm{H} 2)$ it was $36.9 \%(32.1 \%$ to $41.9 \%)$, and homozygous individuals for the $\mathrm{H} 2$ allele it was $5.0 \%$ (3.7\% to $6.4 \%$ ).

Figure 1 shows the results of all studies included in the present meta-analysis (1305 cases and 1194 controls). The summary odds ratio, under a fixed effect model, shows that individuals homozygous for $\mathrm{Hl}$ were 1.57 times more likely (95\% CI, 1.33 to $1.85 ; \mathrm{p}<0.00001$ ) to develop Parkinson's disease than $\mathrm{H} 2$ carriers $(\mathrm{H} 1 \mathrm{H} 2+\mathrm{H} 2 \mathrm{H} 2)$.

We did identify significant interstudy heterogeneity ( $\mathrm{p}$ value for heterogeneity $\left.\left(\mathrm{P}_{\mathrm{Het}}\right)=0.01\right)$. Likewise, a modest degree of interstudy variability was also observed $\left(\mathrm{I}^{2}=62.1 \%\right)$. However, a sensitivity analysis showed that the study of Farrer et al, ${ }^{7}$ conducted in an isolated Norwegian population, largely accounted for this heterogeneity. This was also the study with the largest odds ratio. Nevertheless, a random effect summary odds ratio that takes into account the intrastudy and interstudy variability resulted in a similar and significant overall estimate (OR 1.71 ( 1.25 to 2.36); $\mathrm{p}=0009)$. When this study ${ }^{7}$ was excluded from the summary 
Table 1 Haplotype and genotype frequency for the present case-control study

\begin{tabular}{llllll}
\hline & $\begin{array}{l}\text { Controls } \\
(\mathbf{n}=513)\end{array}$ & $\begin{array}{l}\text { Total PD } \\
(\mathbf{n}=580)\end{array}$ & $\begin{array}{l}\text { YOPD } \\
(\mathbf{n}=\mathbf{2 6 3})\end{array}$ & $\begin{array}{l}\text { Sporadic PD } \\
(\mathbf{n}=193)\end{array}$ & $\begin{array}{l}\text { FPD } \\
(\mathbf{n}=124)\end{array}$ \\
\hline $\begin{array}{l}\text { Genotype } \\
\text { H1H1 }\end{array}$ & $290(56.5)$ & $377(65)$ & $177(67.3)$ & $125(64.8)$ & $75(60.5)$ \\
H1H2 & $188(36.6)$ & $171(29.5)$ & $69(26.2)$ & $57(29.5)$ & $45(36.3)$ \\
H2H2 & $35(6.9)$ & $32(5.5)$ & $17(6.5)$ & $11(5.7)$ & $4(3.2)$ \\
Allele frequency & & & & & \\
H1 (\%) & 74.9 & 79.7 & 80.4 & 79.5 & 78.6 \\
H2 (\%) & 25.2 & 20.3 & 19.6 & 20.5 & 21.4 \\
\hline
\end{tabular}

Values are $\mathrm{n}(\%)$ unless specified.

FPD, familial Parkinson's disease (index familial cases with one or more relative also affected by Parkinson's disease); PD, Parkinson's disease; YOPD, young onset Parkinson's disease (onset before the age of 45 years)

odds ratio, the individual odds ratios were no longer heterogeneous $\left(\mathrm{P}_{\mathrm{Het}}=0.57\right)$, no interstudy variability was observed $\left(\mathrm{I}^{2}=0 \%\right)$, and the summary odds ratio remained statistically significant (summary OR 1.46 (1.23 to 1.73 ); $\mathrm{p}<0.0001$ ).

The distribution of the odds ratios from individual studies compared with their respective standard errors (funnel plot) was slightly asymmetrical, suggesting moderate positive publication bias (fig 2).

When $\mathrm{Hl}$ carriers $(\mathrm{H} 1 \mathrm{Hl}+\mathrm{H} 1 \mathrm{H} 2)$ were compared with $\mathrm{H} 2 \mathrm{H} 2$ (dominant genetic model), no significant association was observed (summary OR 1.27 (0.98 to 1.82 ); $\mathrm{p}=0.19$ ). Furthermore, when a co-dominant model was evaluated, only the $\mathrm{H} 1 \mathrm{Hl} v \mathrm{H} 2 \mathrm{H} 2$ comparison was significant (summary OR 1.46 ( 1.02 to 2.10$) ; \mathrm{p}=0.04$ ), while heterozygosity for the $\mathrm{Hl}$ allele did not increase the risk of Parkinson's disease (summary OR 0.96 (0.66 to 1.40$) ; \mathrm{p}=0.83$ ). These data support the hypothesis that homozygosity for $\mathrm{Hl}$ (recessive model) increases the risk of Parkinson's disease.

The population attributable risk for the tau variant, for the main comparison of $\mathrm{H} 1 \mathrm{Hl}$ against $\mathrm{H} 2$ carriers, was $24.8 \%$ for all studies combined.

\section{DISCUSSION}

The principal observation of this study, and of the metaanalysis, was that homozygosity for the tau $\mathrm{Hl}$ genotype $(\mathrm{HlHl})$ increased Parkinson's disease risk by 57\% (95\% CI, $33 \%$ to $85 \%$; $\mathrm{p}<0.00001$ ).

Furthermore, as the population frequency of the "at risk" tau-H1Hl genotype in this meta-analysis was $58 \%$ in the

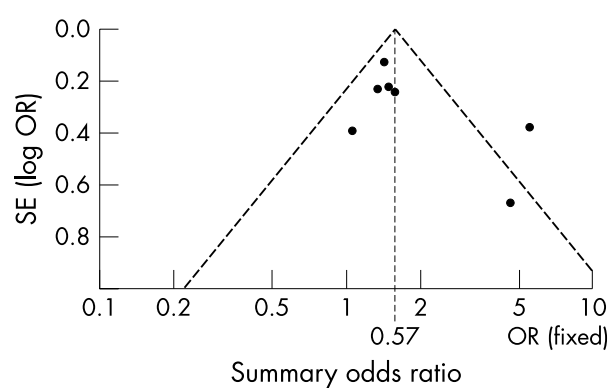

Figure 2 Funnel plot data assessing publication bias in studies evaluating the association between the tau gene and Parkinson's disease. OR, odds ratio.

control samples, the population attributable risk was considerable $(24.8 \%)$. Thus given that the reported incidence of Parkinson's disease ranges between 16 and 19 per 100000 inhabitants per year, ${ }^{23}$ and assuming that our estimate is correct, the tau gene contributes between 2400 and 2900 cases of Parkinson's disease in the United Kingdom each year.

We acknowledge that these findings are difficult to reconcile with the lack of significant tau pathology in idiopathic Parkinson's disease. However, in the normal brain, tau and $\alpha$ synuclein are concentrated in the axon where in principle they could interact. It is possible to hypothesise that such an interaction could influence the propensity of $\alpha$ synuclein to self assemble. In addition, tau aggregation

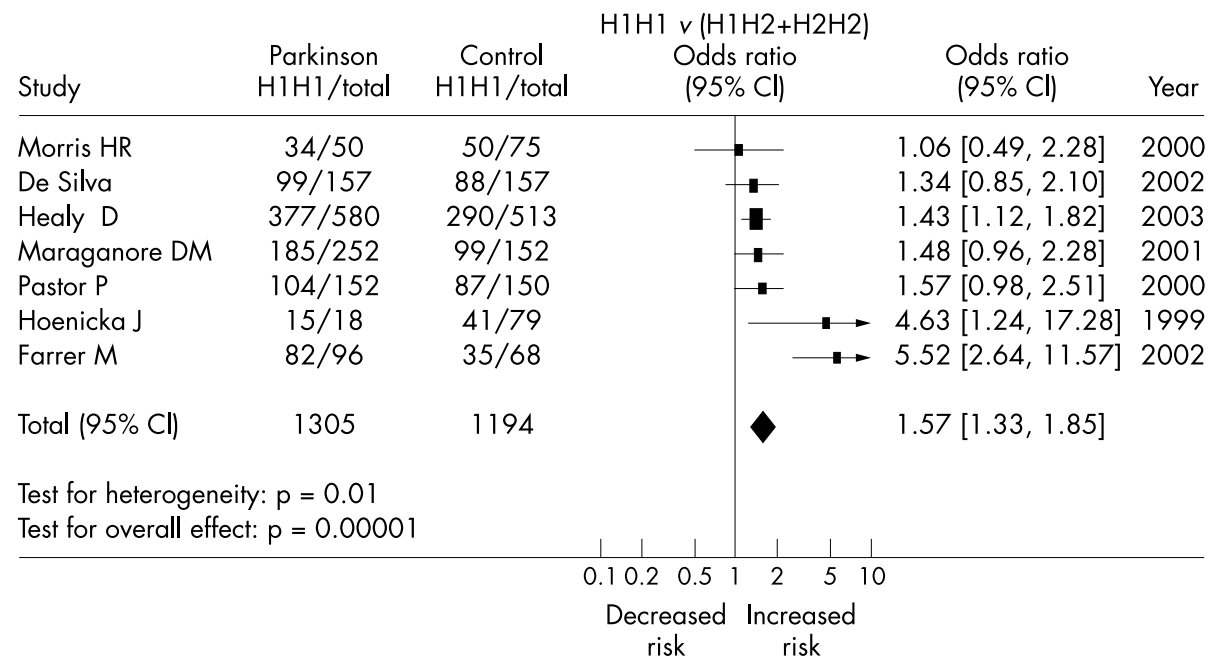

Figure 1 Published association studies between tau and Parkinson's disease. Odds ratio for the outcome compared $\mathrm{H} 1 \mathrm{H} 1$ genotype subjects against $\mathrm{H} 2$ carriers $(\mathrm{H} 1 \mathrm{H} 2$ and $\mathrm{H} 2 \mathrm{H} 2)$. $\mathrm{Cl}$, confidence interval. 
can occur in patients with parkin gene mutations, sometimes in the absence of Lewy bodies, ${ }^{5}$ which raises the possibility that tau dysfunction may also act independently of $\alpha$ synuclein to accelerate dopaminergic neuronal degeneration, and may be a necessary but insufficient factor in the pathogenesis of many cases of Parkinson's disease, especially YOPD.

The genetic architecture of tau is also worth considering. The two extended tau haplotypes, $\mathrm{Hl}$ and $\mathrm{H} 2$, are defined by a small number of polymorphisms that are inherited in complete linkage disequilibrium (LD). If, however, the $\mathrm{Hl}$ haplotype is a misnomer concealing greater genetic variability on the $\mathrm{Hl}$ backbone, or if the "block" of LD-reportedly encompassing the entire tau gene-has different boundaries in different populations, then this may influence the power of the 238 base pair deletion used to define $\mathrm{Hl}$ and $\mathrm{H} 2$ to capture the causal variant in genetically diverse populations.

It has been proposed that the mechanism whereby PSP causes neuronal loss is through the $\mathrm{Hl}$ influencing the excess deposition of the four microtubule binding repeat isoforms of the tau protein. However, given that this histopathological finding is not typically seen in Parkinson's disease, it is possible that another molecular pathway is responsible for the pathogenic effect of the $\mathrm{Hl}$ allele in this condition. If there is more than one subhaplotype within $\mathrm{Hl}$, then a plausible hypothesis would be that one subhaplotype influences PSP pathology while another influences Lewy body pathology. Further work is needed to determine the haplotypic structure of the region around the tau gene, especially given the evolutionary implausibility of just two ancient haplotypes, $\mathrm{Hl}$ and $\mathrm{H} 2$, covering a region of at least $670 \mathrm{~kb}$ in size ${ }^{24}$ and encompassing genes other than the tau, including the recently described saitohin gene. ${ }^{25}$

One perceived problem with case-control design has been the possibility of false positive results from subtle genetic diversity in a given population-that is, population stratification. However, the studies in our meta-analysis used populations of European origin, and recent evidence suggests that, with carefully designed studies and meta-analysis, such populations are unlikely to contain levels of stratification sufficient to cause an inflated number of false positive associations. ${ }^{26} 27$

Positive publication bias, shown by asymmetry in the funnel plot, may have led us to overestimate the tau Hl risk in Parkinson's disease. However, while an empirical assessment of publication bias has shown that in most cases the bias does not affect meta-analyses, ${ }^{28}$ more studies would help refine our estimate of the true effect of the tau $\mathrm{Hl}$.

The observed heterogeneity in this meta-analysis was largely accounted for by a small study in a Norwegian population $^{7}$; the random effect estimation of the summary odds ratio was nevertheless significant, and when this study was excluded from the analysis, the association was still preserved.

\section{Conclusions}

Our new data, as well as the combined data from a metaanalysis, show that subjects homozygous for tau Hl have an increased risk of Parkinson's disease compared with $\mathrm{H} 2$ carriers ( $\mathrm{H} 1 \mathrm{H} 2$ and $\mathrm{H} 2 \mathrm{H} 2$ ). This adds to the growing body of evidence that common genetic variation contributes to the pathogenesis of Parkinson's disease. We propose that the tau gene is implicated in the pathogenesis of this disorder. Whether and how it interacts with other Parkinson's disease genes has yet to be elucidated.

\section{ACKNOWLEDGEMENTS}

We are grateful to the Queen Square Brain Bank for Neurological Disorders, UK, for the provision of the pathological specimens. The work was funded by the Parkinson's Disease Society UK and the Brain Research Trust. We are grateful to William P Gilks for technical assistance. We would also like to thank Karen Shaw for assistance in collecting DNA from patients with Parkinson's disease.

\section{Authors' affiliations}

D G Healy, P M Abou-Sleiman, A J Lees, N W Wood, Department of Molecular Neuroscience, Institute of Neurology, Queen Square, London, UK

N Quinn, K Bhatia, Sobell Department of Motor Neuroscience and Movement Disorders, Institute of Neurology, Queen Square

A J Lees, Reta LilaWeston Institute for Neurological Studies, University of London

J P Casas, A D Hingorani, Centre for Clinical Pharmacology, BHF Laboratories at $\mathrm{UCL}$, London

Competing interests: none declared

\section{REFERENCES}

1 Scott W, Nance M, Watts R, et al. Complete genomic screen in Parkinson disease: evidence for multiple genes. JAMA 2001;286:2239-44.

2 Hutton M, Lendon CL, Rizzu P, et al. Association of missence and 5'-splice site mutations in tau with the inherited dementia FTDP-17. Nature 1998;393:702-5.

3 Ishizawa T, Mattila P, Davies P, et al. Colocalisation of tau and alpha synuclein epitopes in Lewy bodies. I Neuropathol Exp Neurology 2003;62:389-97.

4 Giasson BI, Forman MS, Higuchi M, et al. Initiation and synergistic fibrillization of tau and alpha synuclein. Science 2003;300:636-40.

5 Mori H, Kondo T, Yokochi M, et al. Pathological and biochemical studies of juvenile parkinsonism linked to chromosome 6q. Neurology 1998;51:890-2.

6 De Silva R, Hardy J, Crook J, et al. The tau locus is not significantly associated with pathologically confirmed sporadic Parkinson's disease. Neurosci Lett 2002;330:201-3.

7 Farrer M, Skipper L, Berg M, et al. The tau $\mathrm{H} 1$ haplotype is associated with Parkinson's disease in the Norwegian population. Neurosci Lett 2002;322:83-6.

8 Hoenicka J, Perez M, Perez-Tur J, et al. The tau gene A0 allele and progressive supranuclear palsy. Neurology 1999;53:1219-25.

9 Morris HR, Janssen JC, Bandmann O, et al. The tau gene AO polymorphism in progressive supranuclear palsy and other related neurodegenerative diseases. J Neurol Neurosurg Psychiatry 2000;66:242-5.

10 Pastor $P$, Ezquerra M, Munoz E, et al. Significant association between the tau gene AO/AO genotype and Parkinson's disease. Ann Neurol 2000;47:242-5.

11 Golbe L, Lazzarini A, Spychala J, et al. The tau A0 allele in Parkinson's disease. Mov Disord 2001; 16:442-7.

12 Wiley JA, Lynch T, Skipper L, et al. Genetic variability in tau assessed in Parkinson's disease in Ireland [abstract]. Mov Disord 2002;17(suppl):P513.

13 Maraganore DM, Hernandez D, Singleton AB, et al. Case control study of the extended tau gene haplotype in Parkinson's disease. Ann Neurol 2001;50:658-61.

14 Martin ER, Scott WK, Nance MA, et al. Association of single-nucleotide polymorphisms of the tau gene with late onset Parkinson's disease. JAMA 2001;18:2245-50.

15 Gibb WR, Lees AJ. The relevance of the Lewy body to the pathogenesis of idiopathic Parkinson's disease. J Neurol Neurosurg Psychiatry 1988;51:745-54.

16 Baker M, Litvan I, Houlden H, et al. Association of an extended haplotype in the tau gene with progressive supranuclear palsy. Hum Mol Gen 1999;8:711-15.

17 Mantel N, Haenszel W. Statistical aspects of the analysis of data from retrospective studies of disease. J Nat Cancer Inst 1959;22:719-48.

18 Robin JS, Greenland S, Breslow NE. A general estimator of the variance of the Mantel-Haenszel odds ratio. Am J Epidemiol 1986;124:719-23.

19 DerSimonian R, Laird NM. Meta-analysis in clinical trials. Control Clin Trials 1986;7:177-88.

20 Higgins JP, Thompson SG, Deeks JJ, et al. Measuring inconsistency in metaanalyses. BMJ 2003;327:557-60.

21 Light RJ, Pillemar DB. Summing up: the science of reviewing research. Cambridge, MA: Harvard University Press, 1984.

22 Hughes AJ, Daniel S, Kilford L, et al. Accuracy of clinical diagnosis of Parkinson's disease; a clinico-pathological study in 100 cases. J Neurol Neurosurg Psychiatry 1992;55:181-4.

23 Twelves D, Perkins KS, Counsell C. Systematic review of incidence studies of Parkinson's disease. Mov Disord 2003;18:19-31.

24 Pastor P, Ezquerra M, Tolosa E, et al. Further extension of the $\mathrm{H} 1$ haplotype associated with progressive supranuclear palsy. Mov Disord 2002; 17:550-6.

25 Conrad C, Vianna C, Freeman M, et al. A polymorphic gene nested within an intron of the tau gene: implications for Alzheimer's disease. Proc Natl Acad Sci USA 2002;52:7751-6.

26 Ardlie KG, Lunetta KL, Seielstad M. Testing for population subdivision and association in four case-control studies. Am J Hum Genet 2002;71:304-11.

27 Colhoun HM, McKeigue PM, Davey Smith G. Problems of reporting genetic associations with complex outcomes. Lancet 2003;361:865-72.

28 Sutton AJ, Duval SJ, Tweedie RL, et al. Empirical assessment of effect of publication bias on meta-analyses. BMJ 2000;320:1574-7. 